\title{
The Evaluation System of Sustainable Development Country Based on Multiple Target
}

\author{
Zilong Chen ${ }^{1, a}$, Yuhan Wang ${ }^{2, b}$, Yu Zhao ${ }^{3, c}$ and Pengbo Wang ${ }^{4, c}$ \\ ${ }^{1}$ School of Electronic Engineering, Chongqing University, Chongqing 400044, China; \\ ${ }^{2}$ School of Software Engineering, Chongqing University, Chongqing 400044, China; \\ ${ }^{3}$ School of Telecommunications Engineering, Chongqing University, Chongqing 400044, China. \\ ${ }^{4}$ School of Hong Shen, Chongqing University, Chongqing 400044, China. \\ a1986759901@qq.com, b973230301@qq.com, '80323904@qq.com, ${ }^{\text {d }} 1083545956$ @qq.com
}

Keyword: multiple target, sustainable development, evaluation system

\begin{abstract}
This paper has divided a country into two systems: economic system, environmental system. On the basis of Development evaluation system from United Nations and our analysis, we determined first grade metrics of sub-systems. Then, based on first metrics, we specified second grade metrics and defined the method of calculating first grade metrics to make first grade metrics is positive to sustainable development of sub-systems. After getting specific metrics, we built Linear Evaluation Model for each sub-system. Then based on data from World Bank, we chose 130 countries who have complete data set to calculate the weight of each first grade metric by using entropy method and MATLAB. The specific weights we got are $(0.2449,0.1331,0.1175,0.0998,0.2625,0.1422),(0.2566,0.1615,0.2292,0.1165,0.2362)$. The Static Comprehensive Evaluation Model is the sum of the Linear Evaluation Model of each sub-system. Based on the definition of sustainable development and our metrics, we get overall evaluation model, we can easily find the difference between different developing countries, so ,it can be used give one country advice to become more sustainable by the metric system.
\end{abstract}

\section{Build overall model}

\subsection{The evaluation equation to whole system}

In this paper, one country involve three part: economic sub-system, environmental sub-system, it is obvious that every sub-system is important for one country's development, environmental sub-system represents the potential development and competitiveness in future, economic sub-system represents one country's immaterial influence in different areas now. So we can use these three sub-system analyze one country's sustainable development.

$$
\mathrm{P}=\mathrm{PE}+\mathrm{PO}
$$

Where, P denotes National Sustainable Development Index, PE denotes Economic Sustainable Development Index, PO denotes Environmental Sustainable Development Index

\subsection{Determine influence of different sub-system}

Step1: calculate Standardized matrix

We use Standard-Deviation Method to construct Standardized matrix:

$$
P_{i j}=\frac{A_{i j}-A_{j(\max )}}{A_{j(\max )}+A_{j(\min )}}
$$

Where, $A_{i j}$ denotes ith system index of the country, $A_{j(\min )}$ denotes the minimum index of all countries. $A_{j(\max )}$ denotes the maximum index of all countries.

Step2: calculate entropy of each sub-system index 


$$
E_{j}=-\frac{1}{\ln (N)} \sum_{i=1}^{n} p_{i j} \ln \left(p_{i j}\right)
$$

Step3: calculate the sub-system index redundancy

$$
g_{j}=1-e_{j}
$$

Step4: Determine the weights of each sub-system

$$
\alpha_{j}=\frac{g_{j}}{\sum_{i=1}^{n} g_{j}}
$$

\section{Determine Metrics system}

For metrics related to economic influence are too many, we divide economic metrics into different first grade metrics, however, the first grade metrics can involve irrelevant metrics, so we divide the first grade metrics into different second metrics, then the total economic influence of the countries can be calculated step by step.

\subsection{Calculate first grade metrics}

\subsection{1 analyze economic system}

* Meaning of second grade metrics:

\begin{tabular}{|l|l|}
\hline symbol & meaning \\
\hline$E_{11}$ & value of per capita GDP \\
\hline$E_{12}$ & the growth rates of per capita GDP \\
\hline$G_{\mathrm{e}}$ & Exports of goods and services \\
\hline$G_{\mathrm{i}}$ & Imports of goods and services \\
\hline$E_{31}$ & Central government debt \\
\hline$E_{32}$ & ODA \\
\hline$R$ & Alternative and nuclear energy \\
\hline$E_{\text {output }}$ & Energy production \\
\hline$E_{\mathrm{in}}$ & Energy use \\
\hline$E_{51}$ & Strength of energy production \\
\hline$E_{52}$ & Energy consumption per unit GDP \\
\hline$E_{53}$ & $\begin{array}{l}\text { Energy use (kg of oil equivalent per } \\
\text { capita) }\end{array}$ \\
\hline$E_{61}$ & Rate of waste recycling \\
\hline$E_{62}$ & $\begin{array}{l}\text { harmful waste management(not } \\
\text { including solid waste) }\end{array}$ \\
\hline$E_{63}$ & solid waste management \\
\hline
\end{tabular}

* Calculate first grade metrics

we can calculate first grade metrics as follow: 


\begin{tabular}{|l|l|}
\hline symbol & equation \\
\hline Economic performance & $P_{E 1}=a_{1} \frac{G}{G_{\text {max }}}+a_{2} G_{i}$ \\
\hline Trade & $P_{E 2}=a_{1} \frac{G_{e}}{G_{i-1}}$ \\
\hline Finance & $P_{E 3}=-\left(a_{5} F_{1}+a_{6} F_{2}\right)$ \\
\hline Resource consumption & $P_{E 1}=-\left(a_{31} \frac{E_{31}}{E_{32(\max )}}+a_{32} \frac{E_{32}}{E_{32(\max )}}\right)$ \\
\hline Trade & $E_{51}=\frac{E_{\text {output }}}{E_{\text {in }}}$ \\
\hline Waste emission & $P_{E 5}=-\left(a_{51} \frac{E_{51}}{E_{51(\max )}}+a_{52} \frac{E_{52}}{E_{52(\max )}}+a_{53} \frac{E_{53}}{E_{53(\max )}}\right.$ \\
\hline Energy consumption & $P_{E 5}=-\left(a_{61} \frac{E_{61}}{E_{61(\max )}}+a_{62} \frac{E_{62}}{E_{62(\max )}}+a_{63} \frac{E_{63}}{\left.E_{63(\max }\right)}\right.$ \\
\hline
\end{tabular}

\subsubsection{Analyze environmental system}

* Meaning of second grade metrics

\begin{tabular}{|l|l|}
\hline symbol & meaning \\
\hline$O_{11}$ & CO2 emissions \\
\hline$O_{12}$ & Hydroxide emissions \\
\hline$O_{13}$ & PM2.5 \\
\hline$O_{21}$ & Percentage of arable land \\
\hline$O_{22}$ & Agriculture, value added \\
\hline$O_{31}$ & Forest area \\
\hline$O_{32}$ & Forest rent \\
\hline$O_{41}$ & Annual freshwater withdrawals \\
\hline$O_{42}$ & Improved water source, urban \\
\hline$O_{43}$ & Improved water source, rural \\
\hline$O_{51}$ & $\begin{array}{l}\text { Energy use } \text { capg of oil equivalent per } \\
\text { capita) }\end{array}$ \\
\hline$O_{52}$ & Nature Reserve \\
\hline$O_{53}$ & GEF benefits index for biodiversity \\
\hline
\end{tabular}

* Calculate first grade metrics

We can calculate the first grade metrics as follow: 


\begin{tabular}{|l|l|}
\hline symbol & equation \\
\hline Air Quality & $P_{O 1}=-\left(b_{11} \frac{O_{11}}{O_{11(\max )}}+b_{12} \frac{O_{12}}{O_{12(\max )}}+b_{13} \frac{O_{13}}{O_{13(\max )}}\right)$ \\
\hline Agriculture & $P_{O 2}=-\left(b_{21} \frac{O_{21}}{O_{21(\max )}}+b_{22} \frac{O_{22}}{O_{22(\max )}}\right)$ \\
\hline Forest & $P_{O 3}=-\left(b_{31} \frac{O_{31}}{O_{31(\max )}}+b_{32} \frac{O_{32}}{O_{32(\max )}}\right)$ \\
\hline Fresh water & $P_{O 4}=-\left(b_{41} \frac{O_{41}}{O_{41(\max )}}+b_{42} \frac{O_{42}}{O_{42(\max )}}+b_{43} \frac{O_{43}}{O_{43(\max )}}\right)$ \\
\hline Natural ecological system & $P_{O 5}=-\left(b_{51} \frac{O_{51}}{O_{51(\max )}}+b_{52} \frac{O_{52}}{O_{52(\max )}}+b_{53} \frac{O_{53}}{O_{53(\max )}}\right)$ \\
\hline
\end{tabular}

\section{2 weight of economic sub-system}

Based on data from World Bank and above equation, we can get the first metrics and second metrics of different sub-system, three sub-system are ayalyzed as follows:

\subsection{1 the analysis of economic system}

- weight of economic system

* Calculate weight of first grade metrics

$$
\left[\alpha_{1}, \alpha_{2}, \alpha_{3}, \alpha_{4}, \alpha_{5}, \alpha_{6}\right]=[0.2449,0.1331,0.1175,0.0998,0.2625,0.1422]
$$

Where, $\alpha_{\mathrm{i}}$ denotes coefficient of ith first grade metric in economic system.

* Calculate weight of second grade metrics:

$$
\begin{aligned}
{\left[a_{11}, a_{12}\right] } & =[0.4055,0.5945] \\
{\left[a_{31}, a_{32}\right] } & =[0.3821,0.6179] \\
{\left[a_{51} a_{52}, a_{53}\right] } & =[0.2929,0.2641,0.4430] \\
{\left[a_{61}, a_{62}, a_{63}\right] } & =[0.0582,0.025,0.9167]
\end{aligned}
$$

where, $a_{i j}$ denotes coefficient of jth second grade metric in ith first grade metric.

\section{Conclusion}

- Energy assumption and economic performance play an important in first grade metrics, these two factors can conspicuously affect economic development.

- The weight of most metrics is close, only resource consumption have a lower weight, it proves that most metrics are representative.

\subsubsection{The analysis of environmental system}

- weight of environmental system

* Calculate weight of first grade metrics

$$
\left[\beta_{1}, \beta_{2}, \beta_{3}, \beta_{4}, \beta_{5}\right]=[0.2566,0.1615,0.2292,0.1165,0.2362]
$$

Where, $\alpha_{\mathrm{i}}$ denotes coefficient of ith first grade metric in environmental system.

* Calculate weight of second grade metrics:

$$
\begin{gathered}
{\left[b_{11}, b_{12}, b_{13}\right]=[0.0464,0.1231,0.8304]} \\
{\left[b_{21}, b_{22}, b_{23}\right]=[0.4941,0.0393,0.4666]} \\
{\left[b_{31}, b_{32}\right]=[0.7466,0.2534]} \\
{\left[b_{41}, b_{42}\right]=[0.0504,0.0702,0.8754]} \\
{\left[b_{51}, b_{52}, b_{53}\right]=[0.2362,0.5828,0.1810]}
\end{gathered}
$$

where, aij denotes coefficient of jth second grade metric in ith first grade metric. 


\section{Conclusion}

Atmosphere, biology and forest have a larger weight in first metrics, they represent environmental and ecological capacity, respectively. So, the three factors have an obvious influence on ecological development.

As we can see, every first grade metric have close weight, so, these factors are representative.

\section{References}

[1]Samiha Tahseen, Moinul Hossain, Towards City Sustainability: An Updated Framework, IPCBEE,vo1.32(2012)@(2012), IACSIT Press, Singapore

[2] Jack. How to determine regional sustainable development evaluation index, Progress in geography, 1998.6.

[3] World Commission on Environment and Development (WCED).Our Common Future, New York: Oxford University Press, 1987, 8.

[4] Bell, Simon and Stephen Morse. Sustainability Indicators: measuring the immeasurable, Earth scan, London, 2008.

[5] Daly, Herman, Towards some operational principles of sustainable development, Ecological Economics, 2(1990) 1-6.

[6] http://www.nisd.cass.cn/news/602697.htm

[7] http://wenku.baidu.com/view/08eb6f659b6648d7c1c74639.html 